\title{
Research on Design of Weak Current Measurement System Based on I-V Convertor
}

\author{
Zicheng Guo ${ }^{\mathrm{a},{ }^{,}, \text {Guofu Liu }}{ }^{\mathrm{b}}$,Shilin $\mathrm{Wu}^{\mathrm{c}}$ and Mao $\mathrm{Li}^{\mathrm{d}}$ \\ Department of Instrument Science and Technology, National University of Defense Technology \\ Changsha 410073, Hunan China. \\ a413947405@qq.com, ${ }^{b}$ Igflh@163.com, cofkdwsl@sina.com, ${ }^{d}$ limao1993@foxmail.com
}

\begin{abstract}
In this paper, after introducing several methods of measuring weak current, a weak current measurement system is designed which consists of an ultra-low bias current operation amplifier ADA4530 based on the trans-impedance amplifier topology, a secondary amplifier, an eight-order Butterworth low-pass switched-capacitor filter and other modules. Special consideration on the low noise pre-amplifier in practical applications has been proposed particularly. The simulation results of noise characteristics have shown that the system can realize the weak current measurement of $\mu \mathrm{A} \sim \mathrm{pA}$ wide range.
\end{abstract}

Keywords: Weak current, trans-impedance amplifier, noise characteristics, leakage current.

\section{Introduction}

In modern measurements, weak signal detection becomes more and more important. In particular, the measurement of the weak current $\left(10^{-6} \mathrm{~A}\right.$ and below) has a significant role in the field of space particle detection, nuclear radiation detection, life sciences, electrochemistry, and environmental monitoring [1-3]. However, the weak current, which is so small vanishingly that could be annihilated by outside disturb and intrinsic noise in measurement itself, is difficult to be measured. Therefore, we build a smart weak current measurement system that can realize the current measurement of $\mu \mathrm{A} \sim \mathrm{pA}$ wide range, with automatic zero adjustment and range switching function, which is of great significance in the field of weak current detection.

\section{Weak Current Measurement Method}

Weak current can't be measured directly and is usually converted to other quantities and amplified to accomplish the measurement indirectly. The most commonly used methods are the current-to-voltage conversion (IVC) and the current-to-frequency conversion (IFC). IVC includes capacitance integral method and trans-impedance amplifier method. The capacitance integral method requires the charge of accumulation at both ends of the integrating capacitor, which is suitable for the measurement of the mean value of the unstable current, but can't satisfy the requirement in fast change, continuous measurement occasion because of poor real-time performance [4]. The trans-impedance amplifier achieves current-to-voltage conversion through the operation amplifier and feedback resistor, and is real-time measurable. But it requires a larger feedback resistor whose temperature stability is poor, moreover, the input resistance, bias current and other factors of the operation amplifier also affect the measurement precision [5]. IFC is a current-to-frequency convertor circuit [6]. The core part of IFC is a capacitor integrator yet, whose real-time performance is poor in the same way. Furthermore, the complex structure of an IFC makes it difficult to debug. 


\section{Weak Current Measurement System Design}

The weak current measurement system we proposed mainly includes an I-V convertor, a secondary amplifier, a cut-off frequency adjustable low-pass filter, a differential driver, a high-precision analog-to-digital convertor and a microprocessor. Its structure is shown in Fig. 1.

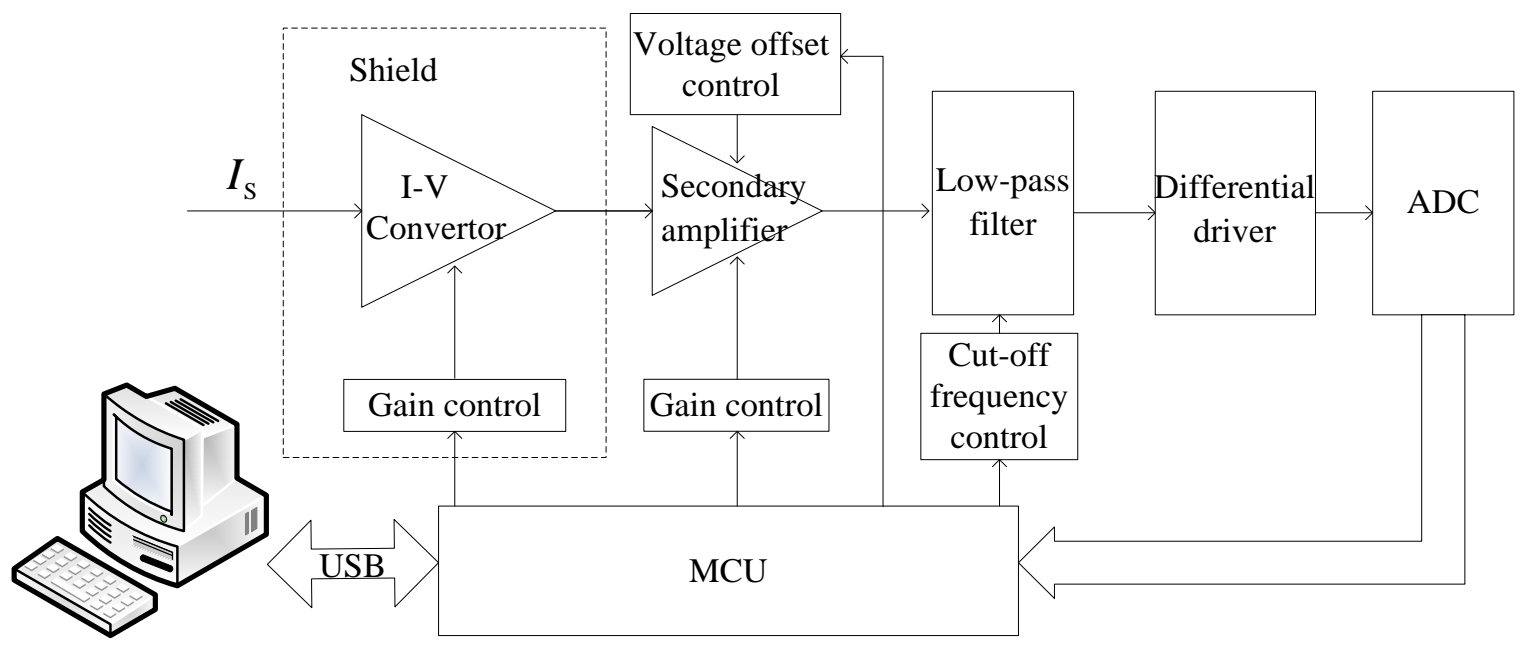

Fig.1 Block diagram of weak current measurement system

The weak current is firstly converted to $\mathrm{mV} \sim \mathrm{V}$ level by the low-noise I-V convertor with the gain controlled by MCU, and then amplified properly further by the secondary amplifier to take full advantage of the dynamic range of the high-precision ADC. The amplified signal voltage comes into low-pass filter in order to reduce noise bandwidth and prevent aliasing when the ADC data acquisition occurs, and the high-precision ADC transmits the collected digital data corresponding to analog voltage to MCU for predispose. Finally, The MCU sends data in real-time to PC through the USB interface for further storage, analysis, processing for obtaining the value of weak current.

\subsection{I-V convertor}

Weak current signal is extremely weak, so the requirements of the background noise generated by measurement system are very strict, otherwise the weak current will be annihilated so that can't be accurately measured. According to the Ferris formula, when the multi-stage amplifier is cascaded, if the first-stage amplifier gain is high enough, the overall noise figure of the system depends primarily on the first stage [7]. So the I-V convertor design determines the system signal-to-noise ratio, measurement accuracy and resolution. As shown before, the IVF and IFC have the advantages and disadvantages. In order to ensure the real-time measurement, we use the trans-impedance amplifier structure in I-V convertor to design the preamplifier. The operation amplifier resorts to the ADI's latest low bias current electrometer amplifier with a bias current of less than 1fA, an offset voltage of typically $8 \mu \mathrm{V}$, a low offset drift of $0.13 \mu \mathrm{V} /{ }^{\circ} \mathrm{C}$, a voltage noise density of $14 \mathrm{nV} / \sqrt{\mathrm{Hz}}$ @10kHz, a current noise density of $0.07 \mathrm{fA} / \sqrt{\mathrm{Hz}} @ 0.1 \mathrm{~Hz}$, input resistance greater than 100T $\Omega$, and integrated guard buffer for isolation of the input pin to prevent leakage on the PCB board leakage, with superior performance, suitable for very low leakage current requirements of the weak current measurement applications [8].

The I-V convertor circuit is shown in Fig. 2. The MCU selects the feedback resistor by controlling the range switch $\mathrm{K} 1, \mathrm{~K} 2, \mathrm{~K} 3$ after analyzing the data obtained from ADC conversion. The value of $R_{\mathrm{f}}=10 \mathrm{G} \Omega, 100 \mathrm{M} \Omega, 1 \mathrm{M} \Omega$ are responding to $1 \mathrm{pA} \sim 100 \mathrm{pA}, 100 \mathrm{pA} \sim 10 \mathrm{nA}, 10 \mathrm{nA} \sim$ $1 \mu \mathrm{A}$ range of current amplification, respectively, so that the output of the $\mathrm{I}-\mathrm{V}$ convertor is $10 \mathrm{mV} \sim$ $1 \mathrm{~V}$, which can facilitate post-processing. As the weak current to be measured converts to voltage directly through the large-value feedback resistor $R_{\mathrm{f}}$, it is necessary to select the resistor with low 
voltage coefficient, low temperature coefficient and high stability, meanwhile a temperature compensation type capacitor with the value about $\mathrm{pF}$ should be parallel on $R_{\mathrm{f}}$ to suppress high frequency noise and compensate the input capacitor of operation amplifier [9]. A relay would be a great choose for feedback resistor selection switch which has high insulation resistance, so as to avoid leakage current. There are many factors should be considered in practical production of this circuit. For instance, placing a $0.1 \mu \mathrm{F}$ ceramic capacitors and a $10 \mu \mathrm{F}$ tantalum capacitors on the operation amplifier power supply pins, respectively, can achieve the greatest degree of suppression of high-frequency power supply noise. Using the coaxial cable as a transmission line can provide a great shied effect and protect the weak current from radiate disturb and noise. Leakage current might contribute to the error source. Fortunately, the ADA4530 integrates an ultra-high performance guard buffer to protect signal current from leakage current. Figure 2 shows the section that should be guarded. In addition, the fluctuations of the input bias current of operation amplifier $I_{\mathrm{B}}$ because of temperature changes directly reflected to the operation amplifier output, which affects the measurement accuracy. The insulation resistance of the materials used to construct circuits is sensitive to moisture. The material adsorbing or absorbing water molecules can produce conductive molecular thin films or change the conductivity of the material. The former is the surface effect that can be suppressed by the guard ring. As to the latter, the circuit can be protected from the effect of localized humidity fluctuation by using the air baffle or shield to restrain the air around the sensitive circuit. So it is necessary to place the I-V convertor in the environment where proper temperature and humidity is obtained and implement appropriate cleaning procedures to suppress the formation of a parallel leakage path caused by contaminants, such as solder flux, body oils, dust and so on.

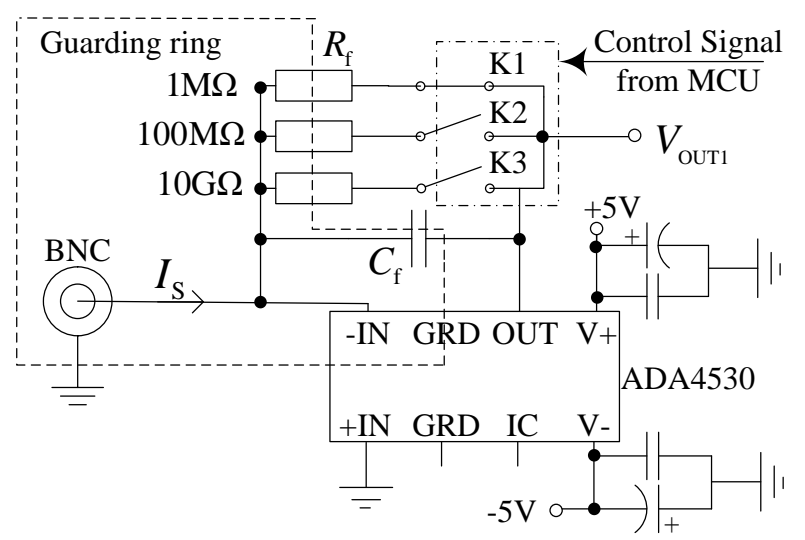

Fig. 2 Schematic diagram of I-V convertor

\subsection{Secondary amplifier}

The preamplifier has converted the weak current into voltage by three segments and improved the signal to noise ratio effectively, which makes the difficulty to design post-processing greatly reduced. As the conversion gain interval of I-V convertor is large, it is necessary to employing a secondary amplifier to get a fine-tune amplification and controllable DC offset voltage. Not only can it compensate for the static error of the previous circuits, but also meet the ADC input voltage requirements and make full use of its dynamic range. The schematic diagram of the secondary amplifier is shown in Fig. 3.

The voltage gain of $\mathrm{U}_{1}, \mathrm{~A}_{1}=1+R_{3} / R_{2}$, in the non-inverting amplifier structure, is automatically changed by adjusting $R_{3}$, which is a digital potentiometer controlled by the MCU according to the data collected by the ADC; $\mathrm{U}_{2}$ is configured in inverting amplifier with adjustable DC offset voltage, which is controlled by the digital potentiometer $R_{6}$. The final calculation:

$$
V_{\text {out3 }}=-V_{\text {out } 1}\left(1+\frac{R_{3}}{R_{2}}\right) \frac{R_{5}}{R_{4}}+V_{\text {set }}\left(1+\frac{R_{5}}{R_{4}}\right)
$$


If we make $R_{4}=R_{5}$, the gain and DC offset voltage can be controlled by the MCU respectively.

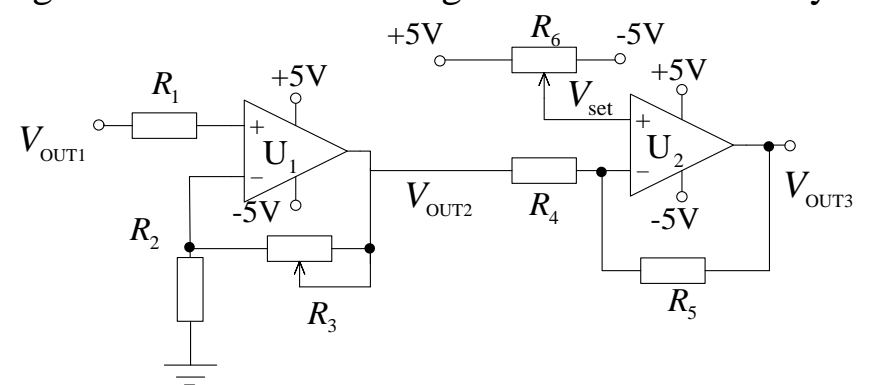

Fig. 3 Schematic diagram of the secondary amplifier

\subsection{Low-pass filter and analog-to-digital convertor}

Low-pass filter can reduce the noise bandwidth to improve the signal to noise ratio further. Moreover it can weaken the disturb generated by power frequency and prevent the ADC sampling from aliasing. The filter in this system uses integrated switching capacitor filter chip MAX291, which is an eighth-order low-pass Butterworth filter, with the largest flat bandwidth response; cut-off frequency $0.1 \mathrm{~Hz} 25 \mathrm{kHz}$ with only an external capacitor or external clock signal needed, easy to adjust. Compared to the traditional R, C active filter, switched capacitor filter is easier to install, without the need for multiple external devices, cut-off frequency adjustment simple.

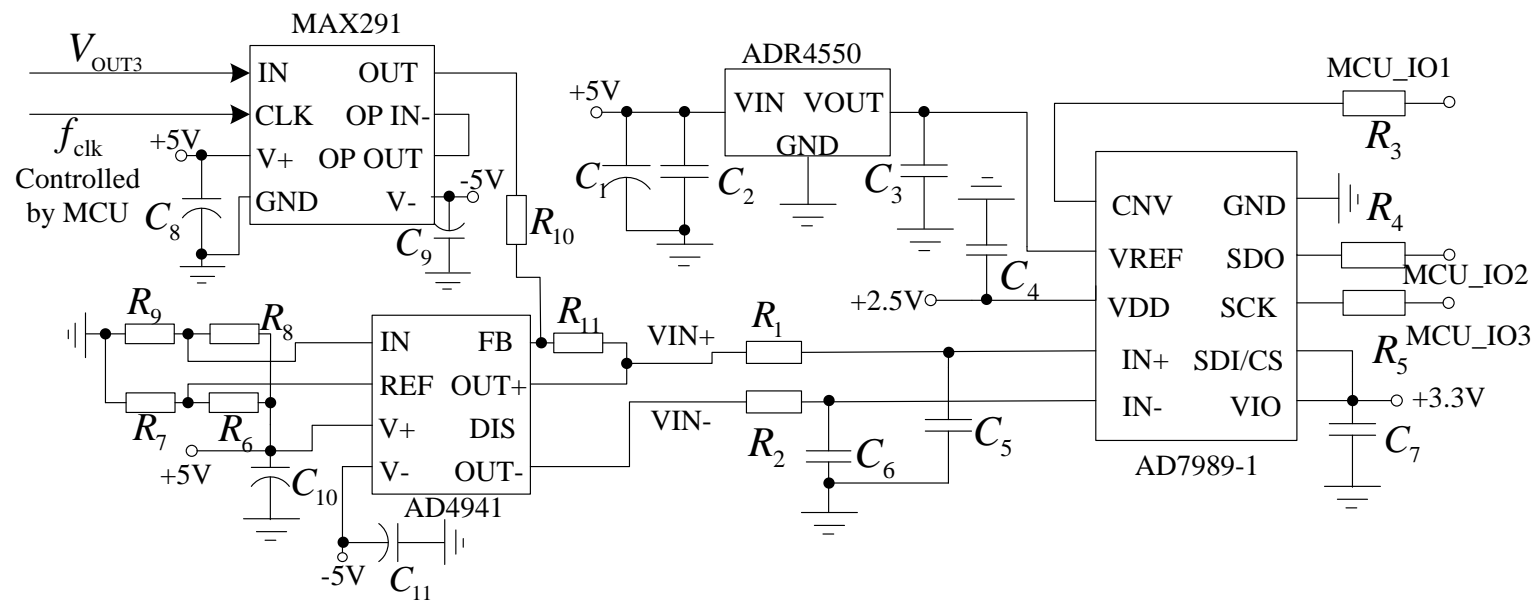

Fig. 4 Schematic diagram of low-pass filter and analog-to-digital conversion

Analog-to-digital convertor is AD7989-1 manufactured by ADI, which is 18-bit, successive approximation, analog-to-digital converters (ADCs) that operate from a single power supply, VDD. It contains a low power, high speed, 18-bit sampling ADC and a versatile serial interface port with a maximum sampling rate of $100 \mathrm{kSPS}$ and only consuming $400 \mu \mathrm{W}$. On the CNV rising edge, the AD7989-1 samples the voltage difference between the IN+ and IN- pins and the voltages on these pins usually swing in opposite phases between $0 \mathrm{~V}$ and VREF. It is widely usually used in data acquisition systems and medical instruments.

The reference voltage of the AD7989-1 is provided by the ADR4550. The ADR4550 is a high accuracy, low noise, low power reference voltage source with an initial error of only $\pm 0.02 \%$ and has extremely high temperature stability to provide accurate reference voltage for the AD7989-1. As shown in Fig. 4, the ADC input is a differential signal obtained from the differential driver AD4941 whose input is the single ended signal derived from the low-pass filter, and the common mode voltage of VIN + and VIN- is half of the VREF.

\section{Simulation and Analysis}

The I-V convertor in the weak current measurement system determines the minimum current that 
can be measured and we use TINA-TI circuit simulation tool for noise characteristics simulation of the I-V convertor. As the post-stage circuit has little effect on the signal-to-noise ratio, the simulation data can be used as a reference for the measurement resolution of weak current measurement system.

By using the noise analysis of TINA-TI, the output noise voltage spectrum density of I-V convertor $e_{\left.\text {n_out }_{\text {outal }}\right)}$ shown in Fig. 5 is obtained. When $R_{\mathrm{f}}=1 \mathrm{M} \Omega, e_{\mathrm{n}_{\text {_out }} \text { (total) }}$ depends predominantly on the equivalent input noise voltage of the operation amplifier, so we will see a curve similar to $1 / \mathrm{f}$ noise, which is larger at lower frequencies. When $R_{\mathrm{f}}=100 \mathrm{M} \Omega$, the resulting noise of operation amplifier $e_{\mathrm{n}}$ and the thermal noise of $R_{\mathrm{f}}$ is contiguous, so they determine the size of $e_{\mathrm{n}_{-} \text {out (total) }}$ together. There is a clear peak at the vicinity of $2 \mathrm{kHz}$, where the initial analysis is that may be caused by the phase lag because of input capacitor, which is similar to the spikes in the curve of AC transfer characteristics. It can be eliminated by increasing $C_{\mathrm{f}}$, but the bandwidth will become narrow, so there is always a tradeoff and compromise we should take. When $R_{\mathrm{f}}=10 \mathrm{G} \Omega$, $e_{\mathrm{n}}$ and $i_{\mathrm{n}}$ of the operation amplifier can be ignored, so the thermal noise of $R_{\mathrm{f}}$ dominates.
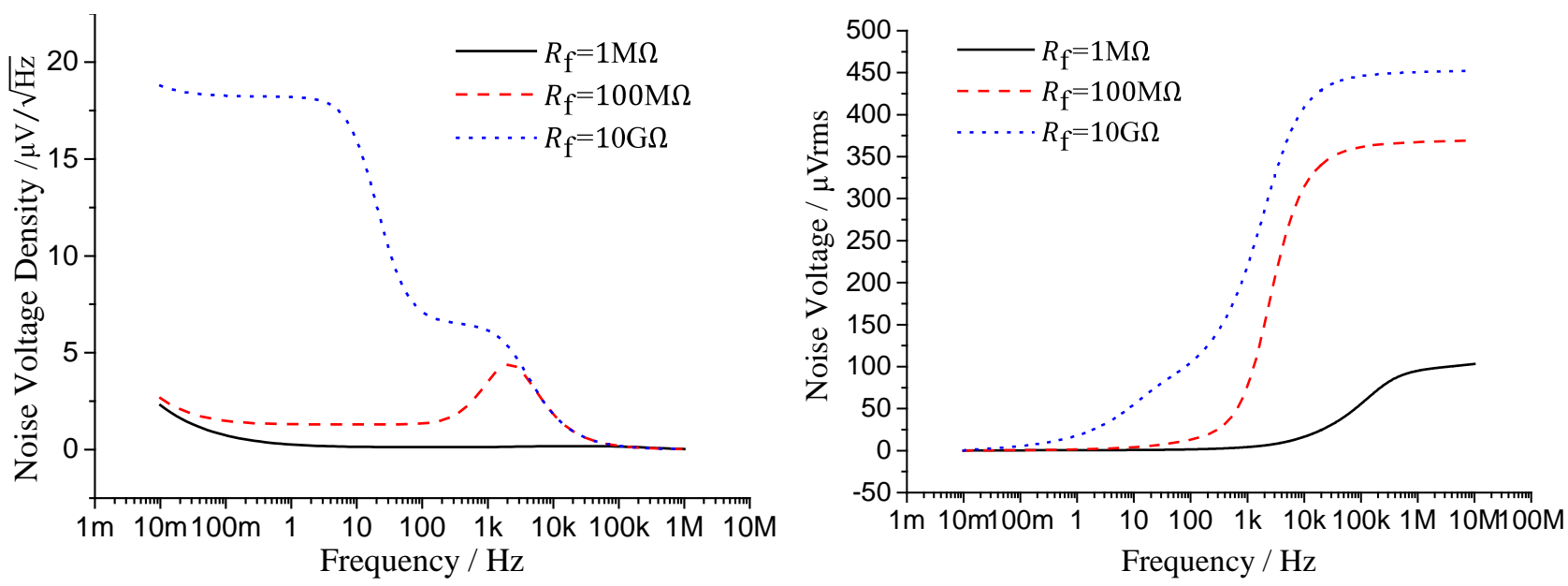

Fig. 5 Output noise voltage spectrum density

Fig. 6 Total output noise voltage vs. frequency

Fig. 6 shows the total output noise of the I-V convertor. The total noise output is the integral of the noise spectrum density in the bandwidth. The wider the bandwidth is, the greater the noise would be. The bandwidth should be controlled according to the frequency range of the signal to be measured. At the same time, we can observe that the total noise is substantially constant after frequency is greater than $100 \mathrm{kHz}$, which is due to $C_{\mathrm{f}}$.

Table 1 Detectable current resolution with different $R_{\mathrm{f}}$

\begin{tabular}{cccc}
\hline$R_{\mathrm{f}}$ & $\begin{array}{c}\text { Expected measurable current } \\
\text { range }\end{array}$ & $\begin{array}{c}\text { Total output noise (rms) } \\
\mathrm{BW}=10 \mathrm{kHz}\end{array}$ & $\begin{array}{c}\text { Minimum measurable current } \\
\text { (10 times than noise) }\end{array}$ \\
\hline $1 \mathrm{M} \Omega$ & $1 \sim 0.01 \mu \mathrm{A}$ & $16.48 \mu \mathrm{V}$ & $164.8 \mathrm{pA}$ \\
$100 \mathrm{M} \Omega$ & $10 \sim 0.1 \mathrm{nA}$ & $314.08 \mu \mathrm{V}$ & $31.41 \mathrm{pA}$ \\
$10 \mathrm{G} \Omega$ & $100 \sim 1 \mathrm{nA}$ & $409.29 \mu \mathrm{V}$ & $0.409 \mathrm{pA}$ \\
\hline
\end{tabular}

Assuming that the amplitude of the current signal to be measured is at least 10 times the amplitude of the noise, the current signal can be effectively amplified and measured. When the bandwidth is $10 \mathrm{kHz}$, the detectable current resolution is obtained from the simulation data of Fig. 10. As shown in Table 1, The minimum measurable current meet the design expectation and the system can realize the conversion and amplification of the weak current in the range of $\mu \mathrm{A} \sim \mathrm{pA}$. 


\section{Summary}

In this paper, the weak current measurement method is briefly introduced, and the I-V conversion method of the trans-impedance amplifier is used as the preamplifier to build the weak current measurement system. In addition, the secondary amplifier, the low-pass filter and the high precision analog-to-digital conversion are also described. The simulation results show that the system that we proposed can meet the requirements of current measurement in the range of $\mu \mathrm{A} \sim \mathrm{pA}$, and it has obvious practicality and engineering value.

\section{Acknowledgements}

This work was financially supported by the National Natural Science Foundation of China under Grand no. 11375264.

\section{References}

[1] Shengjie Lei, Zhiyong Wei, Guoyun Chen, Design of Weak Current Measurement Circuit in Space Particle Detector, J. Journal of Astronustic Metrology and Measurement. 6 (2010), 45-50. [2] Liqian Wei, Shengjie Lei, Meihua Fang, An I-V converter in picoampere current measurement, J. Instrumentation Analysis Monitoring. 3 (2010), 28-31.

[3] Dongsoo Kim, Member, IEEE, Brian Goldstein, Graduate Student Member, IEEE, Wei Tang Noise Analysis and Performance Comparison of Low Current Measurement Systems for Biomedical Applications, J. IEEE TRANSACTIONS ON BIOMEDICAL CIRCUITS AND SYSTEMS. 7 (2013), 52-62.

[4] Shu Hao, Xianguo Tuo, Honghui Wang, Design of Weak Current Measurement with Capacitor-integration Based on STM32, J. Electrieal Measurement \& Instrumentation. 49 (2012), 84-88.

[5] Guiyu Zhang, Xianguo Tuo, Honghui Wang. Comparison and Improvement with the Capacity of C/R Measurement Method of fA Level Current, J. Electrieal Measurement \& Instrumentation. 48 (2011), 8-12.

[6] Guanghua Man, Measurement method of wide range current, C. Proceedings of the 10th National Conference on Electronics \& Nuclear Detection Technology.

[7] Guofu Liu, Jun Yang, Weak signal detection technique, China Machine Press, Beijing, 2014, 54-57.

[8] ADA4530 Data Sheet. http://www.analog.com/ada 4530-1 ?doc= ada4530-1.pdf.

[9] Jun Peng. Measurement of electronic circuit design: analog part. Science Press, Beijing, 2006, 45-48.

[10] Walt Jung, Op amp applications handbook. Posts and Telecom Press, Beijing, 2009, 184-188. 